\begin{tabular}{|c|c|}
\hline Title & The venodilation effects of tapping versus massaging for veni puncture \\
\hline Author(s) & Y asuda, Kae; Sato, Saki; Okada, Kazunori; Y ano, Rika \\
\hline Citation & $\begin{array}{l}\text { Japan journal of nursing science: JJNS, 16(4), 491-499 } \\
\text { https://doi.org/10.1111/jjns.12261 }\end{array}$ \\
\hline Issue Date & $2019-10$ \\
\hline Doc URL & http:/hdl.handle.net/2115/79660 \\
\hline Rights & $\begin{array}{l}\text { This is the peer reviewed version of the following article: Y asuda K, Sato S, Okada K, Y ano R. The venodilation effects } \\
\text { of tapping versus massaging for venipuncture. Jpn J Nurs Sci. 2019;16(4):491-499., which has been published in final } \\
\text { form at https://doi.org/10.1111/jjns.12261. This article may be used for non-commercial purposes in accordance with } \\
\text { Wiley Terms and Conditions for U se of Self-A rchived V ersions. }\end{array}$ \\
\hline Tyре & article (author version) \\
\hline File Information & Y asuda etc 2019.pdf \\
\hline
\end{tabular}

Instructions for use 


\section{The Venodilation Effects of Tapping Versus Massaging for Venipuncture.}

Running title: Comparing two venodilation techniques

Kae Yasuda $\mathrm{RN}^{1}$, Saki Sato $\mathrm{RN}^{2}$, Kazunori Okada MT $\mathrm{PhD}^{3}$, Rika Yano RN $\mathrm{PhD}^{4}$

${ }^{1}$ Graduate School of Health Sciences, Hokkaido University, Hokkaido, Japan

${ }^{2}$ Hokkaido University Hospital, Hokkaido, Japan

${ }^{3}$ Assistant Professor, Faculty of Health Sciences, Hokkaido University, Hokkaido, Japan

${ }^{4}$ Professor, Faculty of Health Sciences, Hokkaido University, Hokkaido, Japan

\section{Correspondence}

Rika Yano, Faculty of Health Sciences, Hokkaido University, Hokkaido University, Kita 12,

Nishi 5, Kita-ku, Sapporo, Hokkaido, 060-0812 Japan.

Phone number: +81 117063323

Fax number: +81 117064911

Email address: r-yano@med.hokudai.ac.jp 


\section{Abstract}

Aim: To compare the effectiveness of tapping and massaging venodilation techniques by evaluating venous cross-sectional area, venous depth, venous palpation score, and questionnaire responses of study participants.

Method: This study had a quasi-experimental design. Between August 2016 and October 2016, healthy adult volunteers ( $\mathrm{n}=30$, mean \pm standard deviation of age: $22.3 \pm 2.2$ years) were enrolled in this study. Three venodilation techniques were evaluated: the application of a tourniquet (Control Group), the application of a tourniquet and tapping of the participant's forearm (Tapping Group), and the application of a tourniquet and massaging of the participant's forearm (Massage Group).

Results: In all three groups, venous cross-sectional areas increased significantly after the application of the venodilation technique. The change ratio of venous cross-sectional area was significantly larger in the Massage Group than in the Control Group. Additionally, 83.3\% of the participants selected massaging as their preferred venodilation technique, stating the technique was comfortable and provided a feeling of relief.

Conclusion: No significant differences were observed between the degrees of venodilation that were achieved using the three investigated venodilation techniques. Nonetheless, massaging was deemed the most effective technique after considering the participants' subjective comments.

\section{Key words}

Massaging, Tapping, Tourniquet, Venipuncture, Venodilation 


\section{INTRODUCTION}

Although venipuncture is used in daily medical practice to withdraw blood or administer intravenous injections, the procedure is invasive and has several risks, such as nerve injury, hematoma, and extravasation. In particular, extravasation of anticancer drugs can cause serious secondary disease, including necrosis (Dougherty, 2002; How, \& Brown, 1998; Mary, 2011). To avoid complications, thick elastic veins should be selected when performing venipuncture, and venodilation should be encouraged. Medical professionals use a variety of techniques to facilitate venodilation, such as applying a tourniquet, asking patients to clench their fists, massaging or tapping patients' forearms, and applying hot compression to patients' forearms (Kuramoto, Watanabe, \& Shinozaki, 2017; Mbamalu, \& Banerjee, 1999; Sabri, Szalas, Holmes, Labib, \& Mussivand, 2013; Roberge, 2004). When venodilation is promoted, venous cross-sectional area (VCS) and diameter increase, while venous depth reduces. Additionally, it is thought that venous palpation becomes possible (Ichimura, Sasaki, Mori, \& Ogino, 2015; Yamagami, Tomita, \& Inoue, 2017).

Several studies have demonstrated the promotion of venodilation via local warming of the forearm, as evaluated through ultrasonographic measurement of VCS (Sasaki et al. 2014; Tokizawa, Tomita, \& Inoue, 2016; Yamagami et al. 2017). In one of these studies, the effect of tourniquet application after local warming was evaluated using prospective, parallel group, singleblinded randomized controlled trial design. The trial results showed that the addition of tourniquet application after local warming increased VCS significantly, as compared with the use of applying a tourniquet alone (Yamagami et al, 2017). However, local warming is a time-sensitive method, as it may cause a low-temperature burn if applied for long durations. In a survey of the venodilation techniques that were actually performed during short peripheral catheter placement, clinical nurses 
in Japan indicated that they attempted to promote venodilation not only by applying a tourniquet or local warming, but also by tapping or massaging the venipuncture area (Kuramoto et al, 2017). Another study investigated the effects of tapping or massaging venodilation techniques by measuring VCS, venous depth, venous elevation, and venous palpation score (VPS). The study included two comparisons. In the first, tourniquet application with tapping was compared with tourniquet application alone. In the second, tourniquet application with massaging was compared with tourniquet application alone. The results indicated that the addition of tapping provided significantly more effective venodilation than tourniquet application alone (Ichimura et al, 2015). However, the study results do not allow direct comparison between the venodilation effects of tapping and massaging because the duration of tourniquet application was different for each technique. Taking this limitation into account, we judged that tapping was not necessarily more effective than massaging, especially considering that tapping can cause pain and stimulation, and could therefore be less comfortable for patients than massaging.

In a study investigating the relationship between phlebotomy experience and intravenous catheterization success, the success rate of vein selection for peripheral intravenous cannulation was higher among nurses who had several years of experience than among novice nurses (Sumitani, \& Watanabe, 2010). In addition, venodilation techniques were used by more expert nurses than novice nurses. These results suggest that proper venodilation is important for successful peripheral intravenous catheterization, and that expert nurses perform various medical procedures with greater accuracy, including venodilation techniques. In a study that investigated the application of venodilation techniques, eight of the 13 expert nurses performed massaging venodilation techniques, rather than tapping or local warming (Masamoto, \& Yano, 2018). We re-analyzed the venodilation techniques of these eight expert nurses and found that they usually performed 
massaging twice per applying a tourniquet. Thus, we hypothesized that massage, which is the technique preferred by expert nurses, may be more effective than tapping for the promotion venodilation. The aim of this study was to compare the venodilation effectiveness of tapping and massaging the forearm by measuring the VPS, VCS, venous depth, and change ratio associated with each technique.

\section{METHODS}

\section{Study design}

This study had a quasi-experimental design. Three venodilation techniques were evaluated: the application of a tourniquet (Control Group), the application of a tourniquet and tapping the participant's forearm (Tapping Group), and the application of a tourniquet and massaging the participant’s forearm (Massage Group). The three groups were compared with each other.

\section{Participants}

Convenience samples of volunteers were recruited. Eligible participants aged 20-29 years were recruited from a national university via posters and flyers between June 2016 and August 2016. All participants were provided oral and written information about this study. Thirty-three participants provided individual informed consent and were included in this study.

The exclusion criteria were (i) undergoing treatment for cardiovascular disease or (ii) receiving treatment for severe skin diseases, wounds, or eczema on the cubital fossa.

\section{Sample Size}


Using G*Power software (version 3.1.9) (G*Power, Heinrich Heine University, Germany) and taking into consideration the results of the studies conducted by Ichimura et al. (2015) and Sasaki et al. (2014), it was calculated 30 participants as the minimum sample size with the following settings: significance level of paired t-test [Control group (only applying a tourniquet) versus Intervention group (applying a tourniquet and other venodilation technique)] of 0.05 ; power of test of 0.80; effect size of 0.50 .

\section{Experimental procedure}

The trials took place at a national university in Japan between August 2016 and October 2016. All interventions and measurements were taken in the same experiment room with a temperature and humidity that were set at the hospital standard for summer (temperature: $22-24^{\circ} \mathrm{C}$, humidity: $45-$ $65 \%)$. We asked all participants to be in a seated position and to keep their forearms on the desk during the experiment. The duration of the wash-out time was set at 10 minutes based on our pilot study.

\section{Supposition of target vein}

To reduce any associated morbidity, medical professionals are recommended to perform venipuncture in the non-dominant arm of the patient (Mary, 2011). In this study, the target vein was the median cubital vein, which tends to be less painful during puncture than other veins (Hori, Miura, Hiromi, Harada, \& Shimada, 2009; Mikuni, Chiba, \& Tonosaki, 2013).

\section{Venodilation procedures}


Figure 1 illustrates each venodilation technique. Each participant was assigned all three venodilation techniques, which were applied in a randomized order with a 10-minute wash-out period between applications. The same researcher performed each technique. To ensure reproducibility, well-trained mid-level and expert nurses provided guidance to the researchers on the venodilation techniques of tapping and massaging. Participants were instructed not to clench their fists.

\section{Applying a tourniquet}

Tourniquets were applied $10 \mathrm{~cm}$ above the target vein (World Health Organization, 2010; Ortega, Selhar, Song, Hansen, \& Peterson, 2008). The application of a tourniquet at $75 \mathrm{mmHg}$ enables the vein to dilate without resulting in unnecessary pain (Kato, \& Mori, 2009a). A rubber-type tourniquet with a scale (tourniquet with scale, TTQ-100-1, TAIYO Instruments INC, Osaka, Japan) was used to ensure the tourniquet was applied at a consistent pressure of approximately 75 mmHg for 30 seconds (Mori et al. 2011). When applying a tourniquet at 20-100 mmHg, VCS almost reaches its maximum value during the 30 seconds, and subsequent increases in VCS are reported to be slight (Sasaki, Murakami, Matsumura, Ichimura, \& Mori, 2012).

\section{Tapping the target vein}

“Tapping” was defined as lightly popping the target vein with the researcher’s right index finger and middle finger. Tapping was performed 10 times during the last 5 seconds of tourniquet application (Ichimura et al. 2015).

Massaging the forearm

“Massaging” was defined as holding the participant's forearm with the researcher's thumb and other fingers and stroking from the wrist to the cubital fossa. Massaging was performed 5 seconds 
after the start tourniquet application, applying eight strokes in 5 seconds and 15 seconds after the start tourniquet application, applying four strokes in 3 seconds (Fig. 2).

\section{Data collection}

In this study, there were six points of data collection: at the start of experiment (T1), after the first venodilation technique (T2), after 10 minutes of rest (T3), after the second venodilation technique (T4), after 10 minutes of rest (T5), and after the third venodilation technique (T6) (Fig. 3).

\section{Participant characteristics}

Participants' characteristics were evaluated, including sex, age, height, weight, body mass index, upper arm circumference (UAC), triceps skinfold thickness (TSF), and body temperature. In the experiment room, participants were asked to sit in a chair with a backrest at a desk and rest for 10 minutes while placing both upper arms on the desk. The participant's dominant arm was used to measure UAC and TSF. To ensure measurements were taken at the same intervention site, the researcher marked the target vein and the position of the tourniquet application.

\section{Venous cross-sectional area (VCS) and depth}

Ultrasonography (SONOS5500 Ultrasound System, 11-3L probe, Koninklijke Philips N.V., Nederland) was performed to measure VCS and venous depth at each point (T1-T6). The crosssectional B-mode image of median cubital vein was obtained, to ensure that the probe was not compressing the vein. VCS $\left(\mathrm{mm}^{2}\right)$ was directly measured by manually tracing the border of the venous wall (Fig.4). Venous depth (mm) was calculated as the vertical distance between the skin surface and the top of the venous circle (Fig.4). In order to confirm the validity and reproducibility 
of the measurement, two researchers conducted measurements. One of the researchers was a Registered Medical Sonographer certified by the Japan Society of Ultrasonics in Medicine, who had substantial experience in ultrasonography.

Skin surface temperature

A thermographic image of the inner forearm and palmar skin surface temperature was taken at each measurement point (the inner forearm: T1-T6, palmar: T1) using thermography (R300 manufactured by NEC of Tokyo, Japan).

Venous palpation score (VPS)

Kato’s VPS (Kato \& Mori, 2009b) was altered and determined using a three-step scale (0: target vein was impalpable, 1: target vein was a little palpable, 2: venodilation of target vein appeared to be sufficiently palpable). The same researcher determined the VPS (T1-T6).

Blood pressure, pulse, and body temperature

Blood pressure, pulse, and body temperature were measured in the dominant arm of the participant (T1-T6).

\section{Questionnaire}

Participants were asked to answer a questionnaire after all interventions (T6). The questionnaire included the following question:

If you could choose a venodilation technique (tapping or massaging) as a patient, which would you choose? 
The participants were asked to respond by writing the name of the technique. Additionally, a space was provided to allow the participants to elaborate on their responses, using their own words (i.e., in an open-ended manner).

\section{Ethical considerations}

Each participant was provided with the following explanations: 1) participation in this study is voluntary and if participation is cancelled, there are no consequences; 2) the data are recorded anonymously and are de-identified; and 3) all data are kept under lock-and-key for over 5 years to maintain confidentiality.

This study was carried out in accordance with the Declaration of Helsinki and was approved by the Ethics Committee of the Graduate School of Health Sciences, Hokkaido University (reference number 16-32).

\section{Data analysis}

The change ratios of VCS and venous depth were calculated as follows: (value after the venodilation technique) / (value before the venodilation technique). Analyses were conducted using InfReC Thermography Studio 5.1 advanced thermal images analysis software (Nippon Avionics of Tokyo, Japan) and SPSS version 22 statistical software (IBM of Armonk, New York, USA). Results (VCS, venous depth, change ratios, the inner forearm skin surface temperature, blood pressure, and pulse) are expressed as the mean \pm standard deviation (SD) and the median. Paired t-tests or Wilcoxon signed-rank tests were performed on the means or medians to compare VCS and venous depth results between the before- and after-intervention groups. Comparisons of VCS or venous depth among the before- and after-intervention groups were performed using one- 
way repeated-measures analysis of variance (ANOVA) and the Bonferroni method, or the Friedman test. VCS and venous depth were classified based on the execution order of the venodilation techniques and were compared using one-way ANOVA. The reliability of values, measured using ultrasonography, was analyzed using intra-rater reliability, ICC (1.1), and interrater reliability, ICC (2.1). The VPS distribution number of each venodilation technique was used as a cross-test.

\section{RESULTS}

Three of the 33 participants were excluded from this study (two participants: measured wrong vein; one participant: scheduling conflict). Thirty healthy student volunteers (15 men and 15 women) participated in this study. The participants ranged in age from 20- to 28-years-old and had normal-range body mass index $\left(18.0 \mathrm{~kg} / \mathrm{m}^{2} \leq \mathrm{BMI}<25.0 \mathrm{~kg} / \mathrm{m}^{2}\right)$ (Table 1$)$.

\section{VCS and associated change ratios}

For each of the three venodilation techniques, VCS increased significantly after technique application (Control Group: $p<.001$, Tapping Group: $p<.001$, Massage Group: $p<.001$ ). However, VCS did not differ significantly among the three techniques, either before or after technique application (Table 2). Evaluating VCS change ratios showed that the Massage Group had a significantly larger value than the Control Group $(p=.006)$. There were no significant differences between VCS change ratio in the Tapping Group and the other groups (Control Group: $p=.101$, Massage Group: $p=1.000)($ Table 3).

\section{Venous depth and associated change ratio}


For each of the venodilation techniques, venous depth became significantly shallower after technique application (Control Group: $p=.023$, Tapping Group: $p=.005$, Massage Group: $p$ $<$.001). However, venous depth did not differ significantly among the three techniques, either before or after technique application (Table 2). Evaluating the change ratios of venous depth did not reveal any significant difference between the three groups (Table 3).

\section{VPS and the associated amounts of change}

None of the study participants had a VPS value that decreased after application of a venodilation technique. Participant VPS responses were divided into three groups based on the amount of change: +0 , VPS remained constant after the venodilation technique; +1 , the venodilation technique was associated with a 1 point increase in VPS; +2, the venodilation technique was associated with a 2 point increase in VPS. For all three techniques, the +1 group included the most participants. When the +1 and +2 groups were combined, the Massage Group was observed to include the highest rate of these responders [Control Group: 21 participants (70.0\%), Tapping Group: 23 participants (76.7\%), Massage Group: 26 participants (86.7\%)]. However, when we conducted a cross test combining the numbers of "+1" and "+2" Group, the difference between the three venodilation group was not statistically significant ( $p=.281$ ). The Massage Group included the highest rate of participants with +2 responses [6 participants (20.0\%)] which was the most palpable level of response (Table 4).

\section{Questionnaire}

The following results were obtained from the participant questionnaire: 
1. If you could choose a venodilation technique (tapping or massaging) as a patient, which would you choose? [n=30; tapping: 5 participants (16.7\%), massaging: 25 participants (83.3\%)].

2. Open-ended responses

Twenty-seven participants answered, of whom 10 responded about Tapping. Six of 10 participants (60\%) felt it was uncomfortable and painful, although 3 participants (30.0\%) felt a venodilation effect and 1 participant (10.0\%) felt comfortable. On the other hand, 17 of the study participant responded about Massaging. Of these 17 participants, 13 (76.5\%) felt comfort, cheer, and warmth; 3 (17.6\%) felt a venodilation effect, and 1 (5.9\%) felt uncomfortable.

\section{Forearm skin surface temperature}

Regarding the inner forearm skin surface temperature, there were no significant differences between the before and after values for any venodilation technique (Control Group: $p=.112$, Tapping Group: $p=.091$, Massage Group: $p=.124$ ). The amount of temperature change was at most $0.1^{\circ} \mathrm{C}$. Additionally, the temperature before the venodilation techniques did not differ significantly among the three groups $(p=.391)$.

\section{Blood pressure and pulse}

For each venodilation technique, application was not associated with a significant difference in blood pressure or pulse (Control Group: systolic blood pressure $p=.058$, diastolic blood pressure $p=.439$, pulse $p=.782$; Tapping Group: systolic blood pressure $p=.381$, diastolic blood pressure $p=.304$, pulse $p=.615$; Massage Group: systolic blood pressure $p=.464$, diastolic blood pressure $p=.807$, pulse $p=.589$ ). Additionally, blood pressure and pulse did not differ 
significantly among the three techniques, as assessed before technique applications (systolic blood pressure $p=.204$, diastolic blood pressure $p=.962$, pulse $p=.637$ ). The maximal observed changes in values were as follows: systolic blood pressure $=3.4 \mathrm{mmHg}$, diastolic blood pressure $=2.4 \mathrm{mmHg}$, and pulse $=2.1$ minutes .

\section{Inter-rater and intra-rater reliability}

VCS and venous depth were measured by two researchers (researchers A and B) and were analyzed by ICC (2.1). The measurements were determined to have high inter-rater reliability (VCS: 0.98, venous depth: 0.97). VCS and venous depth measurements taken mainly by researcher A were also analyzed for intra-rater reliability, which was found to be high (VCS: 0.93, venous depth: $0.83)$.

\section{DISCUSSION}

After the application of each venodilation technique, VCS increased significantly in size. Venous depth after tapping or massaging also significantly decreased (Table 2). Further, the Tapping Group and the Massage Group had larger numbers of participants with +1 or +2 VPS responses, as compared with the Control Group. Therefore, based on these results, venodilation is promoted by the addition of tapping or massaging to tourniquet application.

In addition, the results of the present study suggest that massaging has a positive effect on venodilation. First, VCS change ratio of the Massage Group was significantly larger than that of the Control Group. Second, the Massage Group had the largest number of participants with increases in VPS after the venodilation technique was applied. Finally, massaging showed the highest level of subjective participant satisfaction. However, VCS and venous depth did not differ 
significantly between the Tapping Group and the Massage Group. Thus, tapping cannot be discredited as a venodilation technique, and we consider both methods to be effective.

\section{Tapping}

The specific venodilation mechanism of tapping remains unclear from a physiological standpoint (Mbamalu, \& Banerjee, 1999). When dermises receive a stimulus, such as tapping, antidromic effects of nociceptive C-fibers release chemical mediators, such as calcitonin gene-related peptide (CGRP) and venous endothelium nitric oxide, that promote venodilation (Kindgen-Milles, \& Arndt, 1996; Sabri et al., 2013; Wasner, Baron, \& Janig, 1999). However, in the present study, participants reported more discomfort with tapping than with massaging, commenting that tapping induced pain, an uncomfortable feeling, and a feeling of surprise. Therefore, if medical professionals choose tapping as a venodilation method, they should inform their patients about the potential effects of the technique.

\section{Massaging}

In the present study, a tourniquet was placed $10 \mathrm{~cm}$ above the target vein and then massaging was applied, stroking from the wrist to the cubital fossa. The stroking was divided into two applications because veins have valves, suggesting that the blood pushed upwards by massaging was able to continue to a stasis state at the puncture site near the tourniquet and the valves, thereby promoting venodilation (Mbamalu, \& Banerjee, 1999; Roberge, 2004; Sabri et al., 2013).

In their prior study, Ichimura et al., (2015) compared the combination of tourniquet application and massaging with tourniquet application alone. They reported no significant between-technique differences in VCS or venous depth measurements. These findings differ from the results of the 
present study. We surmised that the method of massaging may have differed between the study of Ichimura et al., (2015) and our own. The massage method used by Ichimura et al., (2015) was extracted from the questionnaire survey responses of nurses who had a wide range of years of experience, including both novices and highly experienced individuals. On the other hand, the massage method used in the present study was selected based on the massage movements employed by expert nurses with high venipuncture success rates. Moreover, Ichimura et al., (2015) explain that, in their study, massage movements were performed 10 times from the wrist to the cubital fossa on the flexor surface during the last 20 of 60 seconds of tourniquet application. In the present study, massaging was performed earlier after the start of tourniquet application, and the massage speed was faster.

Masamoto and Yano (2018) reported that expert nurses perform inspection and palpation between the first and the second massage and assess the target vein. In regard to the duration of tourniquet application and massage, the present study had a shorter application time than that of Ichimura et al., (2015) (present study: 30 seconds, Ichimura et al., (2015): 60 seconds).

The results of the present study suggest that massaging is a venodilation technique that not only achieves a venodilation effect in a shorter amount of time, but also enables assessment of the target vein. In their questionnaire responses, 16 of 17 participants felt that the massaging technique was associated with comfort, cheer, and warmth. During the actual procedure of venipuncture, many patients are expected to feel anxiety and nervousness; therefore, when expert nurses select a target vein, some of them perform massaging to allay anxiety or nervousness (Masamoto, \& Yano, 2018).

\section{Limitations of the study}


The limitations of this study include that the age of the participants were limited to individuals in their twenties, and that one researcher performed all venodilation techniques. Thus, the results of this study are difficult to generalize. In the future, additional studies are needed that verify the the ability to replicate of each venodilation technique. Additional research should also consider the possibility of physical and individual factors that may affect venodilation effects, and verify the venodilation effects in participants who are difficult to intravenously access.

\section{CONCLUSION}

For each of the three investigated venodilation techniques, VCS increased significantly in all three groups and venous depth reduced significantly in Tapping group and Massage group after technique application. The combination of tourniquet application and massaging was associated with larger VCS change ratios than tourniquet application alone. Additionally, VPS increases were observed more commonly after the combination of tourniquet application and massaging, as compared with the combination of tourniquet application and tapping or tourniquet application alone. Further, $83.3 \%$ of the study participants chose massaging as their preferred venodilation technique, stating that the technique was comfortable and provided a feeling of relief. In conclusion, although no significant differences were observed between the degrees of venodilation that were achieved using the three techniques, massaging was deemed to be the most effective in light of the participants' subjective comments.

\section{ACKNOWLEDGEMENTS}


The authors are grateful to Ms. Tsuneko Masamoto for her insights and discussions related to venodilation techniques which is the technique preferred by expert nurses. The authors would like to thank all those who participated in this study.

\section{DISCLOSURE}

The authors declare no conflict of interest.

\section{AUTHOR CONTRIBUTIONS}

This authorship statement confirms that the listed authors meet the authorship criteria and that all authors agree with the content of the manuscript.

K. Y., S. S., and R. Y. designed the study. K. Y., S. S., and K. O. collected the data. K. Y., S. S., and R. Y. analyzed the data. All authors prepared the manuscript and approved the final version for submission.

\section{REFERENCES}

Dougherty, L., (2002). Delivery if intravenous therapy. Nursing Standard. 16, 16, 45-52. dio: 10.7748/ns2002.01.16.16.45.c3134

Hori, M., Miura, M., Hiromi, A., Harada, C., \& Shimada, T., (2009). Morphology of the Topographical Relations between Cutaneous Veins and Nerves in the Human Upper Extremity. Japanese Journal of Nursing Art and Science. 8, 2, 20-28. doi: 10.18892/jsnas.8.2_20 (In Japanese).

How, C., \& Brown, J., (1998). Extravasation of cytotoxic chemotherapy from peripheral veins. European Journal of Oncology Nursing. 2, 1, 51-58. doi: 10.1016/S1462-3889(98)81261-1 
Ichimura, M., Sasaki, S., Mori, M., \& Ogino, T., (2015). Tapping but not massage enhances vasodilation and improves venous palpation of cutaneous veins. Acta Medica Okayama. 69.2, 79-85. doi: org/10.18926/AMO/53336

Kato, A., \& Mori, M., (2009a). The effect of tourniquet for venipuncture on overswelling of the vein Fasten Ratio of tourniquet to upper arm circumference. Japanese Journal of Nursing Art and Science. 8, 10-15. doi: 10.18892/jsnas.8.3_10 (In Japanese).

Kato, A., \& Mori, M., (2009b). The effect of tension of venipuncture tourniquet on overswelling of the vein, and physical factors that influence overswelling. Japanese Journal of Nursing Art and Science. 8, 3, 42-47. doi: 10.18892/jsnas.8.3_42 (In Japanese).

Kindgen-Milles, D., \& Arndt, J.O., (1996). Nitric oxide as a chemical link in the generation of pain from veins in humans. Pain, 64, 139-142.

Kuramoto, N., Watanabe, Y., \& Shinozaki, E., (2017). Survey of Techniques to Induce Vasodilation Performed by Clinical Nurses During Short Peripheral Catheter Placement. Japanese Journal of Nursing Art and Science. 16, 22-35. doi: 10.18892/jsnas.16.0_28 (In Japanese).

Masamoto, T., \& Yano, R., (2018). Characteristics of Observations and Procedures Followed by Expert Nurses for the Selection of the Intravenous Insertion Site for a Short Peripheral Catheter. Japan Academy of Nursing Science (In Japanese) (Posting has been decided).

Mary, A., (2011). Infusion nursing standards of practice. Journal of Infusion Nurse Society. Lippincott Williams \& Wilkins, Philadelphia. 34, S65-74. [Cited 22 Sep 2017.] Available from

URL: https://engage.ahima.org/HigherLogic/System/DownloadDocumentFile.ashx?DocumentFil $\underline{\text { eKey=2238ee0a-c2df-4d1a-affa-f69f2ce41856 }}$ 
Mbamalu, D., \& Banerjee, A., (1999). Methods of obtaining peripheral venous access in difficult situations. The Fellowship of Postgraduate Medicine. 75, 886, 459-462. doi: 10.1136/pgmj.75.886.459

Mikuni, Y., Chiba, S., \& Tonosaki, Y., (2013). Topographical anatomy of superficial veins, cutaneous nerves, and arteries at venipuncture sites in the cubital fossa. Anatomical Science International. 88, 46-57. doi: 10.1007/s12565-012-0169-z

Mori, M., Ichimura, M., Matsumura, Y., Sasaki, S., Murakami, N., Kawasaki, M., \& Hirano, N., (2011). New Tourniquet with Markers for Applying with Appropriate Strength. Bulletin of Faculty of Health and Welfare Science, 18. Okayama Prefectural University, 29-33. doi: $10.15009 / 00000589$

Ortega, R., Sekhar, P., Song, M., Hansen, C. J., \& Peterson, L., (2008) Videos in clinical medicine. Peripheral intravenous cannulation. The new england journal of medicine. 359, e26. doi: 10.1056/NEJMvcm0706789.

Roberge, R.J., (2004). Venodilatation techniques to enhance venepuncture and intravenous cannulation. The Journal of Emergency Medicine. 27, 69-73. doi: 10.1016/j.jemermed.2004.02.011.

Sabri, A., Szalas, J., Holmes, K.S., Labib, L., \& Mussivand, T., (2013). Failed attempts and improvement strategies in peripheral intravenous catheterization. Bio-Medical Materials and Engineering. 23, 93-108. doi: 10.3233/BME-120735

Sasaki, S., Ichimura, M., Murakami, N., Matsumura, Y., Mori, M., \& Ogino, T., (2014). Effect of hot compress on superficial vein of forearm for venipuncture. Japanese Journal of Nursing Art and Science. 12, 14-23. doi: 10.18892/jsnas.12.3_14 (In Japanese). 
Sasaki, S., Murakami, N., Matsumura, Y., Ichimura, M., \& Mori, M., (2012). Relationship between Tourniquet Pressure and a Cross-Section Area of Superficial Vein of Forearm. Acta Medica Okayama. 66, 1, 67-71. doi: 10.18926/AMO/48083

Sumitani, S., \& Watanabe, Y., (2010). Survey of intravenous placement skills with the peripheralshort catheter: comparison of the practice by new nurse midcareer nurse, and experienced nurse. Japan Academy of Nursing Science. 30, 61-69. doi: 10.5630/jans.30.3_61 (In Japanese).

Tokizawa, Y., Tsujimoto, T., \& Inoue, T., (2017). Duration of venodilation for peripheral intravenous cannulation, as induced by a thermal stimulus on the forearm. Biological Research For Nursing. 19, 206-212. doi:10.1177/1099800416651145

Yamagami, Y., Tomita, K., Tsujimoto, T., \& Inoue, T., (2017). Tourniquet application after local forearm warming to improve venodilation for peripheral intravenous cannulation in young and middle-aged adults: A single-blind prospective randomized controlled trial. International Journal of Nursing Studies. J. 72, 1-7. doi: 10.1016/j.ijnurstu.2017.03.009

Wasner, G., Baron, R., \& Janig, W., (1999). Dynamic mechanical allodynia in humans is not mediated by a central presynaptic interaction of A beta-mechanoreceptive and nociceptive C-afferents. Pain, 79, 113-119.

World Health Organization. (2010). WHO guidelines on drawing blood: best practices in phlebotomy. 18-19. [Cited 19 Nov 2018.] Available from URL:

http://apps.who.int/iris/bitstream/handle/10665/44294/9789241599221_eng.pdf;jsessionid =A48C3D7CE038F28B4B1A9FA9DBE6452A ?sequence $=1$ 


\section{FIGURE LEGENDS}

\section{Figure 1 Protocol of venodilation techniques}

Control Group: the application of a tourniquet. Tapping Group: the application of a tourniquet and tapping the patient's forearm. Massage Group: the application of a tourniquet and massaging the patient’s forearm. All participants were performed all venodilation techniques.

\section{Figure 2 Massage technique}

“Massaging” was defined as holding the participant's forearm with the researcher's thumb and other fingers and stroking from the wrist to the cubital fossa.

\section{Figure 3. Study protocol}

: Pint of measurement

$\mathrm{T} 1$, at the start of experiment; T2, after the first venodilation technique; T3, after 10 minutes; T4, after the second venodilation technique; T5, after 10 minutes; T6, after the third venodilation technique.

\section{Figure 4 Venous cross-sectional area and Venous depth}

Skin Surface $=$ Forearm skin surface

VCS (= venous cross-sectional area) was directly measured by manually tracing the border of the venous wall.

Venous depth was calculated as the vertical distance between the skin surface and the top of a venous circle. 
Control Group

Applying a tourniquet for $30 \mathrm{sec}$.

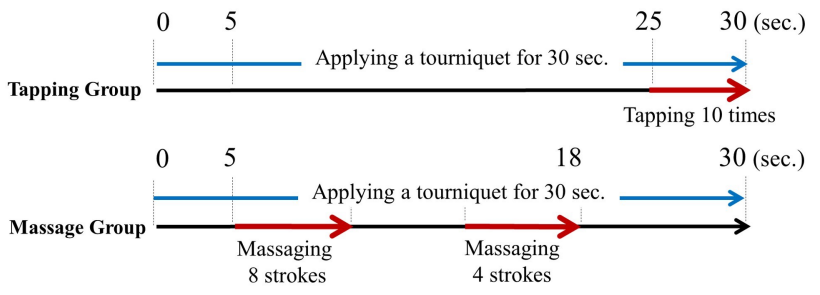


Venous cross section area and Venous depth

Venous palpation score

Blood pressure and Pulse

Skin surface temperature on forearm

Skin surface temperature on palm

Questionnaries

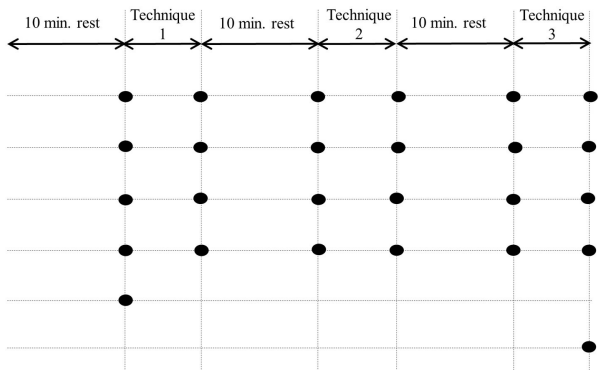




\section{Skin Surface}

\section{Venous depth}

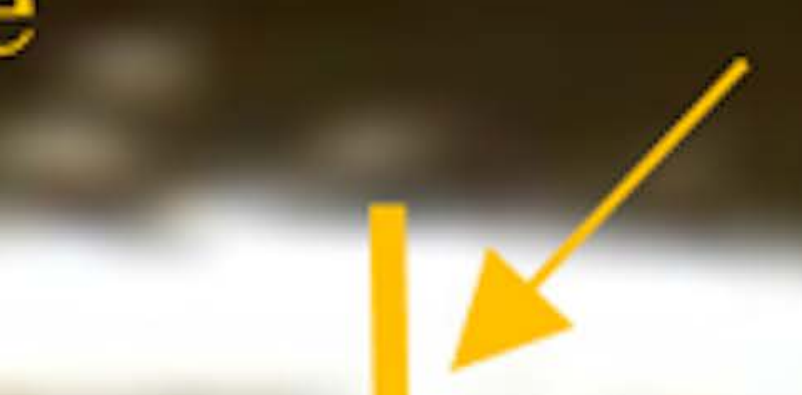

\section{VCS}

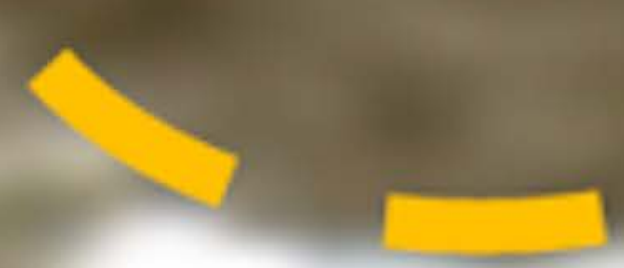


Table 1. Characteristics of the participants ( $\mathrm{N}=30$ : 15 men, 15 women)

\begin{tabular}{lrlr}
\hline Characteristics Item & Mean & \pm & SD \\
\hline Age (years) & 22.3 & \pm & 2.2 \\
Height $(\mathrm{cm})$ & 165.7 & \pm & 8.8 \\
Weight $(\mathrm{kg})$ & 56.5 & \pm & 9.8 \\
BMI $\left(\mathrm{kg} / \mathrm{m}^{2}\right)$ & 20.5 & \pm & 2.4 \\
Systolic blood pressure (mmHg) & 107.6 & \pm & 15.0 \\
Diastolic blood pressure (mmHg) & 64.4 & \pm & 9.3 \\
Pulse (times/minute) & 72.9 & \pm & 10.7 \\
Upper arm circumference (UAC) $(\mathrm{cm})$ & 24.8 & \pm & 3.2 \\
Triceps skinfold thickness (TSF) $(\mathrm{mm})$ & 17.6 & \pm & 5.5 \\
Body temperature $\left({ }^{\circ} \mathrm{C}\right)$ & 36.4 & \pm & 0.3 \\
Skin temperature on the hand $\left({ }^{\circ} \mathrm{C}\right)$ & 32.9 & \pm & 2.0 \\
Skin temperature on the forearm $\left({ }^{\circ} \mathrm{C}\right)$ & 32.6 & \pm & 1.1 \\
\hline
\end{tabular}

Note. $\mathrm{SD}=$ standard deviation. $\mathrm{BMI}=$ Body Mass Index 
Table 2. Comparison VCS and venous depth with each venodilation technique

\begin{tabular}{|c|c|c|c|c|}
\hline \multirow{3}{*}{ Measurement } & \multirow{2}{*}{\multicolumn{2}{|c|}{$\begin{array}{c}\text { VCS }\left(\mathrm{mm}^{2}\right) \\
\text { Mean } \pm \text { SD } \\
\text { Median }\end{array}$}} & \multirow{2}{*}{\multicolumn{2}{|c|}{$\begin{array}{c}\text { Venous depth }(\mathrm{mm}) \\
\text { Mean } \pm \text { SD } \\
\text { Median }\end{array}$}} \\
\hline & & & & \\
\hline & before & after & before & after \\
\hline \multicolumn{5}{|l|}{ Group } \\
\hline \multirow[t]{2}{*}{ Control } & $10.1 \pm 6.8$ & $15.6 \pm 9.7$ & $2.3 \pm 1.0$ & $2.1 \pm 1.1$ \\
\hline & 8.9 & $15.9 * * *$ & 2.2 & $1.7^{*}$ \\
\hline \multirow[t]{2}{*}{ Tapping } & $9.7 \pm 6.2$ & $16.4 \pm 9.2$ & $2.1 \pm 1.0$ & $1.9 \pm 0.9$ \\
\hline & 9.2 & $14.6^{* * *}$ & 1.78 & $1.6^{* *}$ \\
\hline \multirow[t]{2}{*}{ Massage } & $9.3 \pm 5.1$ & $16.4 \pm 8.9$ & $2.1 \pm 0.9$ & $1.8 \pm 0.7$ \\
\hline & 8.6 & $14.8^{* * *}$ & 1.9 & $1.5^{* * *}$ \\
\hline$P$-value & $.924^{\mathrm{a}}$ & $.114^{\mathrm{a}}$ & $.127^{\mathrm{a}}$ & $.275^{\mathrm{a}}$ \\
\hline
\end{tabular}

Note. $\mathrm{SD}=$ standard deviation. $\mathrm{VCS}=$ venous cross-sectional area

Wilcoxon signed-rank test were performed on the means or medians to compare date about VCS and Depth between the before and after intervention groups.

*** represents significant difference $(\mathrm{p}<.001)$, ** represents significant difference $(\mathrm{p}<.01)$, * represents significant difference $(\mathrm{p}<.05)$

${ }^{\text {a }}$ Friedman test. 
Table 3. Comparison the change ratio with each technique

\begin{tabular}{|c|c|c|c|c|}
\hline \multirow[b]{2}{*}{ Measurement } & \multicolumn{2}{|c|}{ VCS } & \multicolumn{2}{|c|}{ Venous depth } \\
\hline & $\begin{array}{l}\text { Mean } \pm \text { SD } \\
\text { 〔Median〕 }\end{array}$ & Min-Max & $\begin{array}{c}\text { Mean } \pm \text { SD } \\
\text { 〔Median` }\end{array}$ & Min-Max \\
\hline \multicolumn{5}{|l|}{ Group } \\
\hline Control & $\begin{array}{c}1.6 \pm 0.4 \\
\lceil 1.6]\end{array}$ & $1.0-2.7$ & $\begin{array}{c}0.9 \pm 0.2 \\
\lceil 0.8]\end{array}$ & $0.6-1.4$ \\
\hline Tapping & $\begin{array}{c}1.8 \pm 0.6 \\
〔 1.8 〕\end{array}$ & $0.9-3.8$ & $\begin{array}{c}0.9 \pm 0.2 \\
\lceil 0.8 〕\end{array}$ & $0.5-1.3$ \\
\hline Massage & $\left.\begin{array}{c}1.9 \pm 0.5 \\
\lceil 1.8]\end{array}\right]$ & $1.1-3.4$ & $\begin{array}{c}0.9 \pm 0.2 \\
\lceil 0.8]\end{array}$ & $0.6-1.5$ \\
\hline$P$-value & $.014^{\mathrm{a}}$ & & $.663^{\mathrm{a}}$ & \\
\hline
\end{tabular}

Note. $\mathrm{SD}=$ standard deviation. VCS = venous cross-sectional area.

${ }^{a}$ Friedman test.

*** represents significant difference $(\mathrm{p}<.001)$, ** represents significant difference ( $\mathrm{p}$ $<.01)$, * represents significant difference $(\mathrm{p}<.05)$ by Wilcoxon signed-rank test with Bonferroni correction. 
Table 4. The number and ratio of people in the changing about VPS before and after techniques.

\begin{tabular}{|c|c|c|c|c|c|c|c|c|c|c|c|c|}
\hline \multirow{3}{*}{ VPS } & \multicolumn{4}{|c|}{+0} & \multicolumn{4}{|c|}{+1} & \multicolumn{4}{|c|}{+2} \\
\hline & \multicolumn{3}{|c|}{ changing } & \multirow[b]{2}{*}{ participants(\%) } & \multicolumn{3}{|c|}{ changing } & \multirow{2}{*}{ participants(\%) } & \multicolumn{3}{|c|}{ changing } & \multirow[b]{2}{*}{ participants(\%) } \\
\hline & before & & after & & before & & after & & before & & after & \\
\hline \multirow[t]{3}{*}{ Control } & 0 & $\rightarrow$ & 0 & 2 (6.7) & 0 & $\rightarrow$ & 1 & 10 (33.3) & 0 & $\rightarrow$ & 2 & 4 (13.3) \\
\hline & 1 & $\rightarrow$ & 1 & 5 (16.7) & 1 & $\rightarrow$ & 2 & 7 (23.3) & & & & \\
\hline & 2 & $\rightarrow$ & 2 & 2 (6.7) & & & & & & & & \\
\hline total & & & & $9(30.0)$ & & & & 17 (56.7) & & & & 4 (13.3) \\
\hline \multirow[t]{3}{*}{ Tapping } & 0 & $\rightarrow$ & 0 & 1 (3.3) & 0 & $\rightarrow$ & 1 & $12(40.0)$ & 0 & $\rightarrow$ & 2 & $3(10.0)$ \\
\hline & 1 & $\rightarrow$ & 1 & 4 (13.3) & 1 & $\rightarrow$ & 2 & 8 (26.7) & & & & \\
\hline & 2 & $\rightarrow$ & 2 & $2 \quad(6.7)$ & & & & & & & & \\
\hline total & & & & 7 (23.3) & & & & 20 (66.7) & & & & $3(10.0)$ \\
\hline \multirow[t]{3}{*}{ Massage } & 0 & $\rightarrow$ & 0 & $0 \quad(0.0)$ & 0 & $\rightarrow$ & 1 & $12(40.0)$ & 0 & $\rightarrow$ & 2 & $6(20.0)$ \\
\hline & 1 & $\rightarrow$ & 1 & $2 \quad(6.7)$ & 1 & $\rightarrow$ & 2 & 8 (26.7) & & & & \\
\hline & 2 & $\rightarrow$ & 2 & $2 \quad(6.7)$ & & & & & & & & \\
\hline total & & & & 4 (13.3) & & & & 20 (66.7) & & & & $6(20.0)$ \\
\hline
\end{tabular}

Note. VPS = venous palpation score.

Score: 0 : Target vein was impalpable, 1 : Target vein was little palpable, 2 : The venodilation of target vein could be palpable enough

We divided the amount of change to three groups (“+0”: the VPS remained after venodilation technique, “+1”: the VPS after venodilation technique become 1 score bigger than the VPS before venodilation technique, “+2”: the VPS after venodilation technique become 2 score bigger than the VPS before venodilation technique. 